"To meet expectations, dental education must do more than render graduating students fit for present purpose."

\title{
Science in dental education
}

The science underpinning the clinical practice of dentistry is set to increase, both in terms of diversity and sophistication. The translation of new knowledge and understanding in relevant aspects of modern molecular biology, let alone other forms of new science - basic, clinical and sociological, may be anticipated to transform traditional approaches to the management of many, if not most forms of oral and dental disease. As is predicted by futurists: If you think recent years have heralded unprecedented change, brace yourself for what is to come - sooner than most of us dare to imagine!

Tomorrow's dentists, will hopefully be able to increasingly delegate to other members of the dental team routine and minimally invasive procedures, including cosmetic enhancements to dental attractiveness, to lead on novel approaches to patient assessment and diagnosis, lifelong treatment planning and the provision of innovative, advanced forms of treatment. The educational environment to suitably prepare tomorrow's dentists must, as a consequence, be firmly rooted in centres of excellence which expose students to experts with special interests and expertise in new and emerging treatment modalities, critical thinking, scholarship and, of increasing importance, scientific rigour and cutting-edge research.

Today's dental students, once qualified, may remain in clinical practice through 2050 and beyond. It is difficult to imagine that existing arrangements for dental education, let alone lifelong learning will fully equip the future members and, in time, leaders of the profession to effectively apply all the new therapies, agents, materials and techniques which will be introduced in the course of their professional lives. Top-up training, over and above revalidation may well become the norm. In the meantime, relevant advances in science, together with new approaches to the scientific underpinning of knowledge should be reinforced in dental education at all levels. Limiting educational exposure to science to the undergraduate programme, let alone the first two or three years of the educational continuum will not be sufficient. In advocating a new approach, it is acknowledged that there will be increasing tensions between new and traditional elements of science in dental education and between scientific knowledge and the skills and competencies fundamental to the practice of dentistry. But, as may be appreciated from reading the works of, for example, G V Black, there is nothing new about striking a balance between the art and science of the clinical practice of dentistry.
With increasing evidence of links between oral and systemic disease and trends towards medical rather than surgical approaches to the management of many oral and dental conditions, medical and dental practitioners should increasingly share the care of patients. To realise this goal, dentists of the future must have opportunity to focus their professional endeavours on the provision of treatments that only they can provide as leaders of the dental team. To ensure parity with medicine in the future, dentistry must stand back and find ways to reinforce and, where necessary, further develop oral healthcare provision as an integral element of holistic, lifelong management of health. In this regard, it is considered important to emphasise the enormous contribution oral health makes to quality of life.

With the inevitable scientific enhancement of dentistry, the need for the scientific enrichment of the delivery of evidencebased care must be addressed. Patients, not to forget the rest of the population, increasingly expect healthcare professionals, including dentists to be able to knowledgably apply new biotechnologies in everyday clinical practice. To meet this expectation, dental education must do more than render graduating students fit for present purpose. Furthermore, to continue to attract some of the brightest and most promising young people into dentistry, dental education must offer more than the opportunity to acquire the knowledge, skills and competencies necessary to practice dentistry as it presently exists.

Within the living memory of the most senior colleagues in the profession, individuals with foresight and determination fought to establish dentistry as an academic discipline worthy of the award of university degrees, up to and including higher doctorates. This legacy must be preserved both through the present period of unprecedented change, and into the future which promises exponential growth in new science and, in turn, metamorphic changes in clinical practice. Tomorrow's leaders of the dental team must have education fit to meet this challenge.

Nairn Wilson

Dean and Head of the King's College London Dental Institute at Guy's, King's College and St Thomas' Hospitals nairn.wilson@kcl.ac.uk

DOI: 10.1038/bdj.2007.234 